University of Wollongong

Research Online

Australian Institute for Innovative Materials -

Papers

Australian Institute for Innovative Materials

2004

Distributed polarizability analysis for para-nitroaniline and meta-nitroaniline: Functional group and charge-transfer contributions

Marc in het Panhuis

University of Wollongong, panhuis@uow.edu.au

R W. Munn

UMIST

P L A Popelier

UMIST

Follow this and additional works at: https://ro.uow.edu.au/aiimpapers

Part of the Engineering Commons, and the Physical Sciences and Mathematics Commons

Research Online is the open access institutional repository for the University of Wollongong. For further information contact the UOW Library: research-pubs@uow.edu.au 


\title{
Distributed polarizability analysis for para-nitroaniline and meta-nitroaniline: Functional group and charge-transfer contributions
}

\author{
Abstract \\ Topological partitioning of electronic properties is used to investigate the polarizability of para- \\ nitroaniline and meta-nitroaniline. The distributed polarizabilities for atoms are combined into total local \\ or generalized distributed contributions for the amino, ring, and nitro functional groups; generalized \\ distributed group contributions have not been calculated before. The local group contributions are \\ transferable between the two molecules only when charge transfer is suppressed, but the generalized \\ distributed contributions prove surprisingly similar in the two molecules, apparently because they treat \\ charge-transfer contributions explicitly.

\section{Keywords} \\ charge, analysis, distributed, polarizability, transfer, contributions, nitroaniline, para, meta, group, \\ functional \\ Disciplines \\ Engineering | Physical Sciences and Mathematics

\section{Publication Details} \\ in het Panhuis, M., Munn, R. W. \& Popelier, P. L. A. (2004). Distributed polarizability analysis for para- \\ nitroaniline and meta-nitroaniline: Functional group and charge-transfer contributions. Journal of \\ Chemical Physics, 120 (24), 11479-11486.
}




\section{AD| $\begin{aligned} & \text { The Journal of } \\ & \text { Chemical Physics }\end{aligned}$}

\section{Distributed polarizability analysis for para-nitroaniline and meta-nitroaniline: Functional group and charge-transfer contributions}

M. in het Panhuis, R. W. Munn, and P. L. A. Popelier

Citation: The Journal of Chemical Physics 120, 11479 (2004); doi: 10.1063/1.1752879

View online: http://dx.doi.org/10.1063/1.1752879

View Table of Contents: http://scitation.aip.org/content/aip/journal/jcp/120/24?ver=pdfcov

Published by the AIP Publishing

\section{Articles you may be interested in}

Local softness, softness dipole, and polarizabilities of functional groups: Application to the side chains of the 20 amino acids

J. Chem. Phys. 131, 044312 (2009); 10.1063/1.3185349

A long-range-corrected density functional that performs well for both ground-state properties and time-dependent density functional theory excitation energies, including charge-transfer excited states

J. Chem. Phys. 130, 054112 (2009); 10.1063/1.3073302

Microscopic calculation of surface-induced second-harmonic generation in crystals of para-nitroaniline J. Chem. Phys. 112, 6757 (2000); 10.1063/1.481251

Resonance Raman intensity analysis of a dicyanovinyl-azaadamantane: Mode-specific reorganization energies for charge-transfer and locally-excited states

J. Chem. Phys. 109, 10958 (1998); 10.1063/1.477792

Excited state polarizabilities in solution obtained by cubic response theory: Calculations on para-, ortho-, and meta-nitroaniline

J. Chem. Phys. 109, 6351 (1998); 10.1063/1.477277

\section{AlP hameded}

Journal of Applied Physics is pleased to announce André Anders as its new Editor-in-Chief 


\title{
Distributed polarizability analysis for para-nitroaniline and meta- nitroaniline: Functional group and charge-transfer contributions
}

\author{
M. in het Panhuis ${ }^{\text {a) }}$ \\ Department of Physics, the University of Texas at Dallas, Richardson, Texas 75083 \\ R. W. Munn and P. L. A. Popelier \\ Department of Chemistry, UMIST, Manchester, M60 1QD United Kingdom
}

(Received 16 January 2004; accepted 30 March 2004)

\begin{abstract}
Topological partitioning of electronic properties is used to investigate the polarizability of para-nitroaniline and meta-nitroaniline. The distributed polarizabilities for atoms are combined into total local or generalized distributed contributions for the amino, ring, and nitro functional groups; generalized distributed group contributions have not been calculated before. The local group contributions are transferable between the two molecules only when charge transfer is suppressed, but the generalized distributed contributions prove surprisingly similar in the two molecules, apparently because they treat charge-transfer contributions explicitly. (C) 2004 American Institute of Physics. [DOI: 10.1063/1.1752879]
\end{abstract}

\section{INTRODUCTION}

Distributing molecular electric multipole moments ${ }^{1}$ and polarizabilities ${ }^{2}$ over the atoms or functional groups that make up a molecule has the practical advantage of accelerating the convergence of multipole expansions for the electrical interactions between molecules. A distribution like this also has the conceptual advantage of helping to interpret the molecular properties in terms of the constituent atoms or functional groups, but this has not been much exploited. One obstacle is the need to define suitably the regions in molecules over which the properties are to be distributed, and the other is the burden of computation. For defining suitable regions, the atoms-in-molecules approach ${ }^{3,4}$ has advantages that have led to its adoption as a method of distribution termed topological partitioning of electronic properties (TPEP),${ }^{5-8}$ and it has been applied in calculating distributed polarizabilities of small molecules. ${ }^{9}$ It has also been used to calculate distributed hyperpolarizabilities in urea, from which the crystal nonlinear optical properties were calculated ${ }^{10}$ and to calculate the distributed polarizability in the water dimer (treated as a supermolecule), which was interpreted in terms of field-induced charge flow along the hydrogen bond between the molecules. ${ }^{11}$ The latter application comes naturally out of the theory of distributed response under the influence of the potential differences that necessarily occur between regions even in a uniform electric field. ${ }^{2}$

Issues of charge movement in a field occur in various areas of importance for applications of molecular materials. One example occurs in molecules for nonlinear optics, where the paradigm for high quadratic nonlinearity is a molecule that consists of an electron donor group attached to an electron acceptor group via a conjugated $\pi$ system, symbolized as $\mathrm{D}-\pi-\mathrm{A}$. Such a molecule will have an admixture of zwitterionic $\mathrm{D}^{+}-\pi-\mathrm{A}^{-}$character in the ground state that will

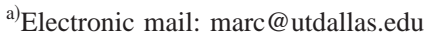

reverse in the first excited state. In a simple two-level sumover-states model, this electronic structure gives a strong contribution to the first hyperpolarizability via the dipole moment change between the two states. Another example is molecular rectification, where the paradigm is a $\mathrm{D}-\sigma-\mathrm{A}$ molecule, this time with a saturated $\sigma$ system acting as a resistive barrier, across which electron flow is easy from donor to acceptor but hard in the reverse direction. ${ }^{12}$ Much effort has been put into trying to achieve rectification in a molecular material and demonstrate unambiguously its molecular origin, and this has also extended to zwitterionic species $\mathrm{D}^{+}-\pi-\mathrm{A}^{-}$as candidate molecules. ${ }^{13} \mathrm{~A}$ third example is the obvious one of molecular conduction, where donor and acceptor groups are not required, but the specific perturbation caused by interaction with the electrode becomes important, as recent calculations show, ${ }^{14-16}$ although insights into conduction through the isolated molecule can also be obtained using TPEP. ${ }^{17}$

To explore some of these issues, we have applied TPEP to the polarizability of the isomeric molecules paranitroaniline (pNA) and meta-nitroaniline (mNA). These serve as model $\mathrm{D}-\pi-\mathrm{A}$ molecules in nonlinear optics (although pNA crystallizes in a centrosymmetric space group that precludes quadratic nonlinearities), and so have been extensively studied. Previous studies of pNA used x-ray diffraction data, ${ }^{18}$ semiempirical and self-consistent field calculations ${ }^{19}$ and the coupled perturbed Hartree-Fock technique $^{20}$ to calculate dipole moments and the first order polarizability, while for mNA these properties were calculated using the density functional theory via the finite-field method. ${ }^{21}$ These papers dealt only with the aggregate molecular dipole moments and polarizabilities, and not the contributions of the functional groups to the molecular properties. We therefore seek to exploit the conceptual advantages of TPEP to interpret the polarizability in terms of contributions from individual $\mathrm{D}, \pi$, and $\mathrm{A}$ groups. Our aim is to provide an interpretation that might also give insights into 
the hyperpolarizability and possible rectifying and conducting behavior-or at least, pointers towards such insights. We implement atoms-in-molecules though the program MORPHY $01^{22}$ as in our previous work, ${ }^{11}$ but with only a small basis set in order to reduce the computational burden. Hence, our results should be regarded as giving not definitive values but rather an illustration of the insights into group response obtainable through distributed polarizability analysis.

\section{METHOD}

Optimized geometries for the molecular structure of $\mathrm{pNA}^{23}$ and for $\mathrm{mNA}^{24,25}$ have been calculated previously. However, for the present work the reported geometries were idealized in order to facilitate the calculations and the comparisons between the two molecules. We replaced the slightly asymmetric ring with a regular hexagonal benzene ring and set the bond angles in the functional groups at $120^{\circ}$. The coordinates were not optimized.

Our method of calculating distributed polarizabilities using the TPEP method has been described previously. ${ }^{9,11}$ It generates a large quantity of information since pNA and mNA each comprise 16 atoms and for each pair of atoms or functional groups the generalized polarizability is a symmetric second-rank tensor with 16 entries, 10 of which are independent. The independent components comprise one chargepotential component, three charge-field or dipole-potential components and six dipole-field components. The calculations are tedious but reasonably straightforward. The wave function is space partitioned and the necessary integrations are performed by MORPHY01. We used the modest basis set $3-21 \mathrm{G}$, which is small enough to handle the large number of two-electron integrals required (involving all real and virtual orbitals) within reasonable computation time. It should nevertheless suffice to permit a semiquantitative investigation of para and meta bonding effects on the distributed polarizability in these nitroanilines, but is too small to give final definitive results.

In order to investigate the role of charge transfer between functional groups, we investigate the polarizability under two separate assumptions, which can be explained with the help of equations from our previous work. ${ }^{11}$ The first assumption is the completely general one that charge flow is allowed within the molecule, while the second is that charge flow is suppressed. The second assumption is made technically feasible by the detailed calculations required in the TPEP method for polarizability, which separate the response into contributions from different regions and separate those contributions into terms that arise from changes in the charge and dipole moment in each region; if charge flow is suppressed, then there are no changes in the charge in each region and the terms from such changes must be excluded, leaving only terms from changes in the dipole moments.

Under the first assumption the molecular polarizability is given by

$$
\alpha_{\alpha \beta}=\sum_{i} \sum_{j}\left(r_{\alpha}^{i} \alpha_{q q}^{i j} r_{\beta}^{j}+r_{\alpha}^{i} \alpha_{q \beta}^{i j}+\alpha_{\alpha q}^{i j} r_{\beta}^{j}+\alpha_{\alpha \beta}^{i j}\right) .
$$

Here $r_{\alpha}^{i}$ is the $\alpha$ component of the vector from the molecular origin to atom $i$, taken as the position of the nucleus. All the quantities $\alpha$ are generalized polarizabilities of various kinds; $q$ as the first subscript refers to a charge and as the second subscript to a potential, while Greek $\alpha$ or $\beta$ as the first subscript refers to Cartesian components of the dipole moment and as the second subscript to Cartesian components of the electric field. The summations run over topological regions, which in the first instance are atomic basins labeled $i$ and $j$, but later will be functional groups. So, for example, $\alpha_{q \beta}^{i j}$ is the polarizability that describes the change of the charge in atomic basin $i$ as a function of the $\beta$ component of electric field in atomic basin $j$.

We describe the molecular polarizability in terms of the functional group contributions, which are combinations of the atom contributions. The obvious functional groups are chosen: the amino group $-\mathrm{NH}_{2}$, the aromatic ring $\mathrm{C}_{6} \mathrm{H}_{4}$, and the nitro group $-\mathrm{NO}_{2}$, corresponding directly to the conventional $\mathrm{D}-\pi-\mathrm{A}$, structure discussed earlier. We assign each atom to a functional group, and rewrite Eq. (1) as

$$
\begin{aligned}
\alpha_{\alpha \beta} & =\sum_{F}\left[\sum_{i \in F}\left(\sum_{G} \sum_{j \in G} r_{\alpha}^{i} \alpha_{q q}^{i j} r_{\beta}^{j}+r_{\alpha}^{i} \alpha_{q \beta}^{i j}+\alpha_{\alpha q}^{i j} r_{\beta}^{j}+\alpha_{\alpha \beta}^{i j}\right)\right] \\
& =\sum_{F}\left[\sum_{i \in F} \alpha_{\alpha \beta}^{i}\right]=\sum_{F} \alpha_{\alpha \beta}^{F},
\end{aligned}
$$

where $F$ and $G$ denote functional groups to which atoms $i$ and $j$ belong. The quantity in the inner parentheses is the total atom dipole-field polarizability $\alpha_{\alpha \beta}^{i}$ for atom $i$ in group $F$, while that in the outer square brackets is the total group dipole-field polarizability $\alpha_{\alpha \beta}^{F}$ for group $F$. For later use, we can also rearrange Eq. (2) as

$\alpha_{\alpha \beta}=\sum_{F} \sum_{G}\left(\sum_{i \in F} \sum_{j \in G} r_{\alpha}^{i} \alpha_{q q}^{i j} r_{\beta}^{j}+r_{\alpha}^{i} \alpha_{q \beta}^{i j}+\alpha_{\alpha q}^{i j} r_{\beta}^{j}+\alpha_{\alpha \beta}^{i j}\right)$,

which allows us to evaluate group generalized polarizabilities by comparison with the analogue of Eq. (1) for groups rather than atoms, namely

$$
\alpha_{\alpha \beta}=\sum_{F} \sum_{G}\left(r_{\alpha}^{F} \alpha_{q q}^{F G} r_{\beta}^{G}+r_{\alpha}^{F} \alpha_{q \beta}^{F G}+\alpha_{\alpha q}^{F G} r_{\beta}^{G}+\alpha_{\alpha \beta}^{F G}\right) .
$$

Here $r_{\alpha}^{F}$ is the $\alpha$ component of the vector from the molecular origin to an origin defined in group $F$ and $\alpha_{q q}^{F G}$, etc., are the group generalized polarizabilities. These can be obtained from Eq. (3) by introducing atom vector components $d_{\alpha}^{i}$ relative to the group origin via $r_{\alpha}^{i}=r_{\alpha}^{F}+d_{\alpha}^{i}$, expanding out products and collecting terms. The coefficients of the group vectors can then be used to identify the group generalized polarizabilities as

$$
\begin{aligned}
& \alpha_{q q}^{F G}=\sum_{i \in F} \sum_{j \in G} \alpha_{q q}^{i j}, \\
& \alpha_{q \beta}^{F G}=\sum_{i \in F} \sum_{j \in G}\left(\alpha_{q q}^{i j} d_{\beta}^{j}+\alpha_{q \beta}^{i j}\right), \\
& \alpha_{\alpha q}^{F G}=\sum_{i \in F} \sum_{j \in G}\left(d_{\alpha}^{i} \alpha_{q q}^{i j}+\alpha_{\alpha q}^{i j}\right),
\end{aligned}
$$


TABLE I. Dipole moments $\mu$ and functional group contributions for pNA and mNA; values in a.u.

\begin{tabular}{|c|c|c|c|c|c|c|c|c|}
\hline \multirow{2}{*}{$\begin{array}{l}\text { Functional } \\
\text { group }\end{array}$} & \multicolumn{4}{|c|}{ pNA } & \multicolumn{4}{|c|}{ mNA } \\
\hline & $\mu_{L}$ & $\mu_{M}$ & $\mu_{N}$ & $\mu$ & $\mu_{L}$ & $\mu_{M}$ & $\mu_{N}$ & $\mu$ \\
\hline $\mathrm{NH}_{2}$ & -7.404 & 0.000 & -0.000 & 7.404 & -6.934 & 1.665 & -0.000 & 7.130 \\
\hline $\mathrm{C}_{6} \mathrm{H}_{4}$ & 2.552 & 0.135 & 0.010 & 2.565 & 5.742 & 3.876 & -0.013 & 6.929 \\
\hline $\mathrm{NO}_{2}$ & 25.095 & 0.002 & -0.003 & 25.095 & 13.365 & -18.486 & -0.013 & 22.812 \\
\hline Total & 20.242 & 0.136 & 0.008 & 20.242 & 12.174 & -12.945 & -0.027 & 17.771 \\
\hline
\end{tabular}

$$
\alpha_{\alpha \beta}^{F G}=\sum_{i \in F} \sum_{j \in G}\left(d_{\alpha}^{i} \alpha_{q q}^{i j} d_{\beta}^{j}+d_{\alpha}^{i} \alpha_{q \beta}^{i j}+\alpha_{\alpha q}^{i j} d_{\beta}^{j}+\alpha_{\alpha \beta}^{i j}\right) .
$$

Under the second assumption, all charge flow is suppressed, and the molecular dipole moment and its constituent atomic dipole moments change only through local changes in the dipole moments, with no contributions from changes in the charges the atoms carry. This implies that all polarizabilities with any subscript $q$ are set to zero, in which case the molecular polarizability reduces to

$$
\hat{\alpha}_{\alpha \beta}=\sum_{i}\left(\sum_{j} \alpha_{\alpha \beta}^{i j}\right)=\sum_{i} \hat{\alpha}_{\alpha \beta}^{i},
$$

or in terms of group polarizabilities to

$$
\begin{aligned}
\hat{\alpha}_{\alpha \beta} & =\sum_{F} \sum_{G}\left(\sum_{i \in F} \sum_{j \in G} \alpha_{\alpha \beta}^{i j}\right)=\sum_{F}\left(\sum_{G} \hat{\alpha}_{\alpha \beta}^{F G}\right) \\
& =\sum_{F} \hat{\alpha}_{\alpha \beta}^{F} .
\end{aligned}
$$

In these equations, the atomic dipole-field polarizabilities $\alpha_{\alpha \beta}^{i j}$ are the same as in Eqs. (1)-(3), whereas the polarizabilities with a circumflex in Eq. (10) are charge-flow suppressed analogs of those in Eqs. (2) and (4). Equation (9) shows that with charge flow suppressed, the molecular polarizability reduces to a sum of distributed atomic dipole-field polarizabilities $\alpha_{\alpha \beta}^{i j}$, while Eq. (10) shows that it can also be expressed as a sum of charge-flow suppressed group generalized polarizabilities $\hat{\alpha}_{\alpha \beta}^{F G}$ or a sum of charge-flow suppressed total group dipole-field polarizabilities $\hat{\alpha}_{\alpha \beta}^{F}$. However, despite the suppression of charge-flow contributions the polarizabilities $\alpha_{\alpha \beta}^{i j}$, and $\hat{\alpha}_{\alpha \beta}^{F G}$ are still nonlocal in the sense that a field at one atom or group can induce a local dipole moment in another, as the double superscripts indicate. Comparing the results under the two assumptions allows us to show how field-induced charge transfer affects the total group polarizabilities $\alpha_{\alpha \beta}^{F}$ and the molecular polarizabilities of the two nitroaniline molecules, and hence, explore para and meta bonding effects on donor-acceptor behavior.

In this paper dipole moments and polarizabilities are quoted in atomic units (a.u.). The a.u. of dipole moment is equal to $e a_{0}$, with $e$ the proton charge and $a_{0}$ the Bohr radius, so that 1 a.u. $=8.478358 \times 10^{-30} \mathrm{C} \mathrm{m}$, which equals $2.5417485 \mathrm{D}$. The atomic unit of polarizability is equal to $4 \pi \epsilon_{0} a_{0}^{3}$, with $\epsilon_{0}$ the permittivity of free space $(0.1648778$ $\times 10^{-40} \mathrm{~F} \mathrm{~m}^{2}$ ). In electrostatic units $4 \pi \epsilon_{0}$ is equal to unity and 1 a.u. can be expressed as $0.1481847 \AA^{3}$.

\section{RESULTS}

\section{A. Dipole moments}

To obtain initial insights into group properties, we have calculated the dipole moments, which are shown in Table I for the two molecules, with their decomposition into functional group contributions. We use conventional $L, M, N$ axes, with the long $L$ axis defined by the amino nitrogen and the ring center, the medium $M$ axis perpendicular to it in the ring plane, and the normal $N$ axis perpendicular to the plane (see Fig. 1). The dipole moment vectors necessarily lie in the molecular $L M$ plane, but the TPEP group results show some deviations from zero for the out-of-plane $N$ components and for the in-plane $M$ components in pNA that should be zero by symmetry. These deviations and similar deviations of the total from that calculated directly, are attributable to cumulative errors from the integrations over atomic basins. The molecular dipole moment is smaller in mNA than in pNA, as expected from the geometry if one assumes comparable contributions from the groups. In pNA, the groups share the molecular symmetry, and hence they have $M$ components that are zero, to the same accuracy as the $N$ components. The net dipole moment is dominated by the nitro group dipole, with a small contribution from the ring group, and an opposing contribution from the amino group. In mNA, the groups have nonzero $L$ and $M$ components. The magnitude of the nitro group dipole is reduced by some $10 \%$ compared with
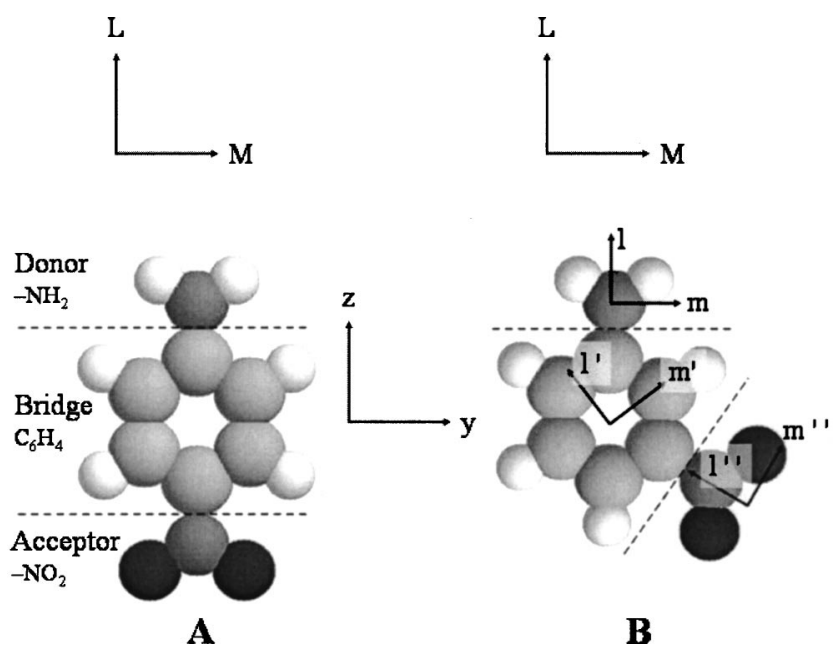

FIG. 1. Picture of para-nitroaniline (a) and meta-nitroaniline (b) with the functional groups shown in the $L, M, N$ axis system; $l m, l^{\prime} m^{\prime}$, and $l^{\prime \prime} m^{\prime \prime}$ indicate group (local) axis systems for the functional groups in metanitroaniline. 
TABLE II. Total group polarizability components $\alpha_{\alpha \beta}^{F}$ and molecular polarizability components $\alpha_{\alpha \beta}$ in $L, M, N$ axes and the corresponding average polarizabilities $\bar{\alpha}=1 / 3 \operatorname{Tr} \alpha$, all with charge flow allowed; values in a.u.

\begin{tabular}{|c|c|c|c|c|c|c|c|c|}
\hline \multirow[t]{2}{*}{ Group } & \multicolumn{4}{|c|}{ pNA } & \multicolumn{4}{|c|}{ mNA } \\
\hline & 43.93 & 0 & 0 & \multirow{3}{*}{$\bar{\alpha}^{\mathrm{NH}_{2}}=17.08$} & 33.17 & -0.79 & 0 & \multirow{3}{*}{$\bar{\alpha}^{\mathrm{NH}_{2}}=13.50$} \\
\hline \multirow[t]{3}{*}{ Amino, $\mathrm{NH}_{2}$} & 0 & 5.64 & 0 & & -8.62 & 5.67 & 0 & \\
\hline & 0 & 0 & $1.68 /$ & & 0 & 0 & 1.67 & \\
\hline & 31.41 & 0.05 & 0.01 & \multirow{3}{*}{$\bar{\alpha}^{\mathrm{C}_{6} \mathrm{H}_{4}}=33.98$} & 40.62 & 4.69 & 0 & \multirow{3}{*}{$\bar{\alpha}^{\mathrm{C}_{6} \mathrm{H}_{4}}=38.05$} \\
\hline \multirow[t]{3}{*}{ Ring, $\mathrm{C}_{6} \mathrm{H}_{4}$} & 0.02 & 57.06 & 0 & & 9.83 & 60.18 & 0 & \\
\hline & 0 & 0 & 13.46 & & 0 & 0 & 13.34/ & \\
\hline & 35.12 & -0.01 & 0 & \multirow{3}{*}{$\bar{\alpha}^{\mathrm{NO}_{2}}=19.45$} & 23.17 & -6.07 & 0 & \multirow{3}{*}{$\bar{\alpha}^{\mathrm{NO}_{2}}=17.18$} \\
\hline \multirow[t]{2}{*}{ Nitro, $\mathrm{NO}_{2}$} & 0.02 & 19.94 & 0 & & -3.39 & 25.00 & 0 & \\
\hline & 0 & 0 & 3.28 & & 0 & 0 & $3.36 /$ & \\
\hline \multirow{3}{*}{ Molecule } & / 110.46 & 0.05 & 0.01 & \multirow{3}{*}{$\bar{\alpha}=70.51$} & 96.96 & -2.18 & & \multirow{3}{*}{$\bar{\alpha}=68.72$} \\
\hline & 0.05 & 82.64 & 0.01 & & -2.18 & 90.84 & 0 & \\
\hline & 0.01 & 0.01 & 18.42 & & 0 & 0 & 18.37 & \\
\hline
\end{tabular}

pNA, but the magnitude of the ring dipole increases significantly in compensation, while the magnitude of the amino group dipole remains essentially unaffected.

These results show that there is no immediate way to interpret the pNA and mNA dipole moments in terms of group moments. For one thing, the ring is not a passive spectator but an active participant, though with a modest role. Nevertheless, it appears possible that since the magnitude of the dipole moment for the nitro group decreases from pNA to mNA while that for the ring increases, suitable transformation of these moments from pNA to mNA might reproduce the total dipole moment. This proves not to be the case. If the nitro group dipole moment from pNA is transformed to the nitro group axes $l^{\prime \prime} m^{\prime \prime}$ in mNA, its $L$ and $M$ components are 12.6 and -21.7 a.u. The $M$ component is already larger than the total $M$ component in mNA, and the $M$ component from the ring in pNA cannot reduce the total as it does in mNA. This is consistent with the valence-bond idea that charge transfer should be less efficient within mNA than within pNA because one cannot write a normal resonance structure that transforms an electron from the amino group to the nitro group in mNA, whereas one can in pNA.

These results show that even in the favorable case of isomers, the concept of a transferable group dipole moment is not quantitatively sustainable. This is already known for sets of chemically distinct molecules. For example, the dipole moment of pNA itself is not the sum of those for nitrobenzene and aniline, and similarly the dipole moment of aminobenzonitrile is not the sum of those for aniline and benzonitrile in the ground state. ${ }^{26}$

\section{B. Polarizabilities}

Table II shows the total group polarizabilities $\alpha_{\alpha \beta}^{F}$ and the molecular polarizabilities $\alpha_{\alpha \beta}$ with charge flow allowed. The total group polarizabilities are not symmetric under interchange of Cartesian components $\alpha$ and $\beta$, as noted previously for total atomic polarizabilities and for total molecular polarizabilities within a dimer, ${ }^{11}$ although together they must yield the proper symmetric total molecular polarizability.
The out-of-plane $N N$ components are too low, a characteristic problem with calculations on aromatic molecules that is to be expected for the rather small basis set we use. Table II shows that when charge flow is allowed the mean group polarizabilities differ rather little between pNA and mNA - by only a few percent, with a correspondingly modest difference between the total polarizabilities-though there are larger differences between individual components. Similar observations were made previously. ${ }^{27}$

Table III shows the total group polarizabilities $\hat{\alpha}_{\alpha \beta}^{F}$ and the molecular polarizabilities $\hat{\alpha}_{\alpha \beta}$ with charge flow suppressed, in which case the mean group polarizabilities differ negligibly between pNA and mNA, though again with some larger differences between individual components. The outof-plane $N N$ components, which arise solely from response within atoms, are not affected by charge flow between atoms and so must be the same for both molecules, but even the others are very close.

Table IV shows the group generalized polarizabilities for pNA calculated from Eqs. (5)-(8) taking the group origin as its center of mass, and Table $\mathrm{V}$ shows the same quantities for mNA. The results show the full symmetry $\alpha_{\lambda \mu}^{F G}=\alpha_{\mu \lambda}^{G F}$ (where $\lambda, \mu=q, L, M, N)$ characteristic of generalized polarizabilities but lacking in group polarizabilities, as already noted; some components are zero for pNA because of its higher symmetry. Direct comparisons between components for the two molecules are not easy, because of the difference in symmetry and because the aromatic ring and the nitro group are differently oriented relative to the $L, M, N$ axes. Despite this, there are considerable similarities. For the amino group, where the axes are oriented in the same way for both molecules, corresponding components $\alpha_{\lambda \mu}^{\mathrm{NH}_{2}, \mathrm{NH}_{2}}$ for pNA and mNA differ by at most $6 \%$, and by less than $1 \%$ for the $L L$ component. This is much better agreement than for the corresponding components $\alpha_{L L}^{\mathrm{NH}_{2}}$ of the total group polarizabilities with charge flow allowed (see Table II), although the charge-flow suppressed $\hat{\alpha}_{L L}^{\mathrm{NH}_{2}}$ agree well (see Table III). Other pairs of corresponding components also resemble one 
TABLE III. Total group polarizability components $\hat{\alpha}_{\alpha \beta}^{F}$ and molecular polarizability components $\hat{\alpha}_{\alpha \beta}$ in $L, M$, $N$ axes and the corresponding average polarizabilities $\overline{\hat{\alpha}}=1 / 3 \operatorname{Tr} \hat{\alpha}$, all with charge flow suppressed; values in a.u.

\begin{tabular}{|c|c|c|c|c|c|c|c|c|}
\hline \multirow{4}{*}{$\begin{array}{l}\text { Group } \\
\text { Amino, } \\
\mathrm{NH}_{2}\end{array}$} & \multicolumn{4}{|c|}{ pNA } & \multicolumn{4}{|c|}{ mNA } \\
\hline & 1.26 & 0 & 0 & & 1.27 & 0.01 & 0 & \\
\hline & 0 & 1.29 & 0 & $\widehat{\hat{\alpha}}^{\mathrm{NH}_{2}}=1.41$ & 0 & 1.30 & 0 & $\overline{\hat{\alpha}}^{\mathrm{NH}_{2}}=1.41$ \\
\hline & 0 & 0 & $1.68 /$ & & 0 & 0 & $1.67)$ & \\
\hline \multirow{3}{*}{$\begin{array}{l}\text { Ring, } \\
\mathrm{C}_{6} \mathrm{H}_{4}\end{array}$} & 6.96 & 0.02 & 0 & \multirow{3}{*}{$\overline{\hat{\alpha}}^{\mathrm{C}_{6} \mathrm{H}_{4}}=9.10$} & 7.23 & 0 & 0 & \multirow{3}{*}{$\overline{\hat{\alpha}}^{\mathrm{C}_{6} \mathrm{H}_{4}}=9.11$} \\
\hline & 0 & 6.87 & 0 & & 0.10 & 6.76 & 0.10 & \\
\hline & 0 & 0 & $13.46 /$ & & 0 & 0 & $13.34)$ & \\
\hline \multirow{3}{*}{$\begin{array}{l}\text { Nitro, } \\
\mathrm{NO}_{2}\end{array}$} & 1.78 & -0.01 & 0 & \multirow{3}{*}{$\overline{\hat{\alpha}}^{\mathrm{NO}_{2}}=2.41$} & 2.07 & 0.17 & 0 & \multirow{3}{*}{$\overline{\hat{\alpha}}^{\mathrm{NO}_{2}}=2.44$} \\
\hline & 0.01 & 2.18 & 0.01 & & 0.16 & 1.88 & 0.16 & \\
\hline & 0 & 0 & $3.28 /$ & & 0 & 0 & 3.36 & \\
\hline \multirow{3}{*}{ Molecule } & 9.99 & 0.01 & 0 & \multirow{3}{*}{$\overline{\hat{\alpha}}=12.92$} & 10.58 & 0.26 & 0 & \multirow{3}{*}{$\overline{\hat{\alpha}}=12.96$} \\
\hline & 0.01 & 10.34 & 0.01 & & 0.26 & 9.94 & 0.26 & \\
\hline & 0 & 0 & 18.42 & & 0 & 0 & 18.37 & \\
\hline
\end{tabular}

another, but the agreement is less striking the more the groups differ from amino and the components differ from $q$ or $L$.

We have also investigated transforming the group generalized polarizabilities for mNA to the local group axes shown in Fig. 1, with the results given in Table VI. One might expect that group polarizabilities would be more transferable in group axes, but this proves not to be the case here. The only components that change on transformation are those involving $L$ or $M$, but for the local polarizabilities $(F=G)$ the off-diagonal $L M$ components are very small and the diagonal $L L$ and $M M$ components differ significantly. As a result, any transformation serves only to induce larger offdiagonal components and to increase the differences from the corresponding components for pNA. At the same time, the transformation cannot change $\alpha_{N N}^{\mathrm{NO}_{2}, \mathrm{NO}_{2}}$, and hence, affords no further insight into why this out-of-plane component differs so much between mNA and pNA when only their inplane structures differ. More work on other molecules is required to investigate the effect of the choice of axes on the transferability of group polarizabilities between molecules.

\section{DISCUSSION AND CONCLUSIONS}

We have used TPEP to calculate the distributed dipole moments and the static distributed polarizability for pNA and mNA, the largest molecules hitherto analyzed in this way, albeit with only a small basis set. Our basic calculations give atomic quantities, but we have combined the atom polarizabilities to give polarizabilities of two sorts for the $-\mathrm{NH}_{2}$,

TABLE IV. Group generalized polarizabilities for pNA. Each matrix gives the components

$$
\left(\begin{array}{cccc}
\alpha_{q q}^{F G} & \alpha_{q L}^{F G} & \alpha_{q M}^{F G} & \alpha_{q N}^{F G} \\
\alpha_{L q}^{F G} & \alpha_{L L}^{F G} & \alpha_{L M}^{F G} & \alpha_{L N}^{F G} \\
\alpha_{M q}^{F G} & \alpha_{M L}^{F G} & \alpha_{M M}^{F G} & \alpha_{M N}^{F G} \\
\alpha_{N q}^{F G} & \alpha_{N L}^{F G} & \alpha_{N M}^{F G} & \alpha_{N N}^{F G}
\end{array}\right) .
$$

\begin{tabular}{|c|c|c|c|c|c|c|c|c|c|c|c|c|}
\hline \multirow[t]{2}{*}{ Group } & \multicolumn{4}{|c|}{$F=\mathrm{NH}_{2}$} & \multicolumn{4}{|c|}{$F=\mathrm{C}_{6} \mathrm{H}_{4}$} & \multicolumn{4}{|c|}{$F=\mathrm{NO}_{2}$} \\
\hline & 1.060 & 0.000 & 0 & 0.525 & -0.922 & 0.000 & 0.000 & -1.337 & -0.139 & 0 & 0 & 0.030 \\
\hline \multirow{3}{*}{$G=\mathrm{NH}_{2}$} & 0.000 & 1.653 & 0 & 0.000 & 0.000 & 0.037 & -0.000 & 0.000 & -0.000 & -0.005 & 0 & 0.000 \\
\hline & 0 & 0 & 6.443 & 0 & -0.000 & 0 & -0.752 & 0.000 & 0 & 0 & -0.052 & 0 \\
\hline & 0.525 & 0.000 & 0 & 2.758 & -0.425 & -0.000 & 0.000 & -0.404 & -0.101 & 0 & 0 & 0.027 \\
\hline \multirow{4}{*}{$G=\mathrm{C}_{6} \mathrm{H}_{4}$} & -0.922 & 0.000 & -0.000 & -0.425 & 1.996 & -0.000 & -0.004 & -0.117 & -1.073 & -0.000 & -0.008 & -0.154 \\
\hline & 0.000 & 0.037 & 0 & -0.000 & -0.000 & 13.533 & 0.002 & 0.004 & -0.000 & -0.110 & 0.003 & -0.001 \\
\hline & -0.000 & -0.000 & -0.752 & 0.000 & -0.004 & 0.002 & 60.445 & 0.033 & -0.000 & 0.001 & -2.628 & -0.009 \\
\hline & -1.337 & 0.000 & 0.000 & -0.404 & -0.117 & 0.004 & 0.033 & 45.779 & 1.435 & 0.002 & 0.036 & 0.535 \\
\hline \multirow{4}{*}{$G=\mathrm{NO}_{2}$} & -0.139 & -0.000 & 0 & -0.101 & -1.073 & -0.000 & -0.000 & 1.435 & 1.212 & 0.000 & -0.000 & 0.130 \\
\hline & 0 & -0.005 & 0 & 0 & -0.000 & -0.110 & 0.001 & 0.002 & 0.000 & 3.394 & -0.000 & -0.000 \\
\hline & 0 & 0 & -0.052 & 0 & -0.008 & 0.003 & -2.628 & 0.036 & -0.000 & 0.000 & 22.617 & -0.000 \\
\hline & 0.030 & 0.000 & 0 & 0.027 & -0.154 & -0.001 & -0.009 & 0.535 & 0.130 & -0.000 & -0.000 & 4.120 \\
\hline
\end{tabular}


TABLE V. Group generalized polarizabilities for mNA in molecular axes. Each matrix gives the components

$$
\left(\begin{array}{cccc}
\alpha_{q q}^{F G} & \alpha_{q L}^{F G} & \alpha_{q M}^{F G} & \alpha_{q N}^{F G} \\
\alpha_{L q}^{F G} & \alpha_{L L}^{F G} & \alpha_{L M}^{F G} & \alpha_{L N}^{F G} \\
\alpha_{M q}^{F G} & \alpha_{M L}^{F G} & \alpha_{M M}^{F G} & \alpha_{M N}^{F G} \\
\alpha_{N q}^{F G} & \alpha_{N L}^{F G} & \alpha_{N M}^{F G} & \alpha_{N N}^{F G}
\end{array}\right) .
$$

\begin{tabular}{|c|c|c|c|c|c|c|c|c|c|c|c|c|}
\hline \multirow[t]{2}{*}{ Group } & \multicolumn{4}{|c|}{$F=\mathrm{NH}_{2}$} & \multicolumn{4}{|c|}{$F=\mathrm{C}_{6} \mathrm{H}_{4}$} & \multicolumn{4}{|c|}{$F=\mathrm{NO}_{2}$} \\
\hline & 1.007 & 0.000 & 0.000 & 0.494 & -0.966 & -0.000 & 0.102 & -1.102 & -0.042 & 0.000 & -0.045 & -0.018 \\
\hline \multirow{4}{*}{$G=\mathrm{NH}_{2}$} & 0.000 & 1.639 & -0.000 & 0.000 & -0.000 & 0.034 & -0.000 & 0.000 & -0.000 & -0.005 & -0.000 & -0.000 \\
\hline & 0.000 & -0.000 & 6.485 & -0.005 & 0.011 & 0.000 & -0.914 & -0.034 & -0.011 & 0.000 & -0.044 & -0.071 \\
\hline & 0.494 & 0.000 & -0.005 & 2.751 & -0.450 & -0.000 & 0.129 & -0.224 & -0.045 & 0.000 & -0.039 & -0.010 \\
\hline & -0.966 & -0.000 & 0.011 & -0.450 & 1.996 & 0.001 & 0.869 & 0.363 & -1.028 & -0.000 & 0.154 & -0.021 \\
\hline \multirow{5}{*}{$G=\mathrm{C}_{6} \mathrm{H}_{4}$} & -0.000 & 0.034 & 0.000 & -0.000 & 0.001 & 13.408 & 0.006 & -0.002 & -0.002 & -0.100 & -0.001 & -0.001 \\
\hline & 0.102 & -0.000 & -0.914 & 0.129 & 0.869 & 0.006 & 58.103 & 4.056 & -0.928 & -0.000 & -0.211 & -1.579 \\
\hline & -1.102 & 0.000 & -0.034 & -0.224 & 0.363 & -0.002 & 4.056 & 51.670 & 0.722 & -0.000 & -1.388 & -1.901 \\
\hline & -0.042 & -0.000 & -0.011 & -0.045 & -1.028 & -0.002 & -0.928 & 0.722 & 1.069 & 0.001 & -0.119 & 0.049 \\
\hline & 0.000 & -0.005 & 0.000 & 0.000 & -0.000 & -0.100 & -0.000 & -0.000 & 0.001 & 3.467 & -0.001 & 0.000 \\
\hline \multirow[t]{2}{*}{$G=\mathrm{NO}_{2}$} & -0.045 & -0.000 & -0.044 & -0.039 & 0.154 & -0.001 & -0.211 & -1.388 & -0.119 & -0.001 & 8.878 & 7.851 \\
\hline & -0.018 & -0.000 & -0.071 & -0.010 & -0.021 & -0.000 & -1.579 & -1.901 & 0.049 & 0.000 & 7.851 & 18.012 \\
\hline
\end{tabular}

$\mathrm{C}_{6} \mathrm{H}_{4}$, and $-\mathrm{NO}_{2}$ functional groups. The first sort comprises the total group polarizabilities such as $\alpha_{\alpha \beta}^{F}$, and the second comprises the group generalized polarizabilities such as $\alpha_{q L}^{F G}$. We believe that Eqs. (5)-(8) for the latter quantities in terms of atom polarizabilities are new. Our calculations are therefore the first of this type, and are consistent with Stone's original treatment of distributed polarizabilities valid for an arbitrary partition of the molecule. ${ }^{2}$ Combining the atomic polarizability contributions into functional group ones significantly reduces the quantity of data as well as allowing chemical questions to be addressed. The results show that the total group polarizabilities become equal in magnitude if charge flow is suppressed but differ significantly when it is allowed. However, subdividing the molecular polarizabilities according to Eq. (4) yields group generalized polarizabilities that differ rather little between the molecules, considering that their different symmetries make some differences inevitable. However, transforming these results to local group axes actually increases the differences, perhaps because the transformed axes are still not symmetry axes.

TABLE VI. Group generalized polarizabilities for mNA in group axes. Each matrix gives the components

$$
\left(\begin{array}{cccc}
\alpha_{q q}^{F G} & \alpha_{q l}^{F G} & \alpha_{q m}^{F G} & \alpha_{q n}^{F G} \\
\alpha_{l q}^{F G} & \alpha_{l l}^{F G} & \alpha_{l m}^{F G} & \alpha_{l n}^{F G} \\
\alpha_{m q}^{F G} & \alpha_{m l}^{F G} & \alpha_{m m}^{F G} & \alpha_{m n}^{F G} \\
\alpha_{n q}^{F G} & \alpha_{n l}^{F G} & \alpha_{n m}^{F G} & \alpha_{n n}^{F G}
\end{array}\right) \text {, in }
$$

which $l$ and $m$ denote axes for the relevant group as shown in Fig. 1, viz. $l$ and $m$ for $F, G=\mathrm{NH}_{2}, l^{\prime}$ and $m^{\prime}$ for $F, G=\mathrm{C}_{6} \mathrm{H}_{4}$, and $l^{\prime \prime}$ and $m^{\prime \prime}$ for $F$,

\begin{tabular}{|c|c|c|c|c|c|c|c|c|c|c|c|c|}
\hline \multirow[t]{2}{*}{ Group } & \multicolumn{4}{|c|}{$F=\mathrm{NH}_{2}$} & \multicolumn{4}{|c|}{$F=\mathrm{C}_{6} \mathrm{H}_{4}$} & \multicolumn{4}{|c|}{$F=\mathrm{NO}_{2}$} \\
\hline & 1.007 & 0.000 & 0.000 & 0.494 & -0.966 & -0.000 & 0.102 & -1.102 & -0.042 & 0.000 & -0.045 & -0.018 \\
\hline \multirow{3}{*}{$G=\mathrm{NH}_{2}$} & 0.000 & 1.639 & -0.000 & 0.000 & -0.006 & 0.029 & 0.457 & 0.000 & -0.010 & -0.003 & -0.038 & -0.000 \\
\hline & 0.000 & -0.000 & 6.485 & -0.005 & 0.010 & 0.017 & -0.792 & -0.034 & -0.006 & 0.004 & -0.022 & -0.071 \\
\hline & 0.494 & 0.000 & -0.005 & 2.751 & -0.450 & -0.000 & 0.129 & -0.224 & -0.045 & 0.000 & -0.039 & -0.010 \\
\hline \multirow{4}{*}{$G=\mathrm{C}_{6} \mathrm{H}_{4}$} & -0.966 & -0.006 & 0.010 & -0.450 & 1.996 & -0.434 & 0.753 & 0.363 & -1.028 & -0.077 & 0.133 & -0.021 \\
\hline & -0.000 & 0.029 & 0.017 & -0.000 & -0.434 & 24.577 & -19.350 & -0.002 & -0.805 & 0.048 & -0.184 & -0.001 \\
\hline & 0.102 & 0.457 & -0.792 & 0.129 & 0.753 & -19.350 & 46.934 & 4.056 & -0.462 & -0.127 & -0.047 & -1.579 \\
\hline & -1.102 & 0.000 & -0.034 & -0.224 & 0.363 & -0.002 & 4.056 & 51.670 & 0.722 & -0.000 & -1.388 & -1.901 \\
\hline \multirow{4}{*}{$G=\mathrm{NO}_{2}$} & -0.042 & -0.010 & -0.006 & -0.045 & -1.028 & 0.462 & -0.805 & 0.722 & 1.069 & -0.103 & -0.060 & 0.049 \\
\hline & 0.000 & -0.003 & 0.004 & 0.000 & -0.077 & 0.048 & 0.127 & -0.000 & -0.103 & 7.524 & 2.344 & 0.000 \\
\hline & -0.045 & -0.038 & -0.022 & -0.039 & 0.133 & -0.184 & -0.047 & -1.388 & -0.060 & 2.344 & 4.821 & 7.851 \\
\hline & -0.018 & -0.000 & -0.071 & -0.010 & -0.021 & -0.000 & -1.579 & -1.901 & 0.049 & 0.000 & 7.851 & 18.012 \\
\hline
\end{tabular}
$G=\mathrm{NO}_{2}$; for all groups the axis $n$ coincides with the molecular axis $N$. 
The simplification obtainable with group generalized polarizabilities seems to be achieved because Eq. (4) treats charge transfer explicitly, which is clearly appropriate in exploring donor-acceptor effects. Other work on distributed polarizability also finds that charge transfer is important. In order to address the practical problem that distributed polarizabilities grow rapidly in number with the number of atoms or groups treated, in addition to consisting of numerous components as already indicated, a scheme was devised to localize distributed dipole polarizabilities by a suitable transformation of origin. ${ }^{28}$ The scheme proved workable for a series of hydrocarbons, provided that charge flow throughout the molecule was retained in an essentially unlocalized form. This was later developed into a practical approach for describing the polarizabilities of the $n$-alkanes in an arbitrary conformation. ${ }^{7}$ The important role of charge transfer in small hydrocarbons was deduced previously, ${ }^{29}$ and general considerations show that transfer of charge between atoms or groups is an essential contribution to the polarization process. ${ }^{30}$

The importance of charge-flow effects in polarizability has a further consequence when we try to compare group contributions. The groups here have nonzero net charges, and hence their dipole moments are origin dependent. It also follows that charge flows induced by an external field are described by polarizabilities that are origin dependent, as noted previously. ${ }^{11}$ As calculated, group properties are normally related to the same molecular origin, typically the center of mass, which is not only convenient but also necessary in order to show how they sum to give the molecular properties calculated directly. However, in order to compare group properties in different molecules, it is desirable to relate them to a common origin in the group. Conversely, Table III shows that when charge flow between groups is suppressed, corresponding group polarizabilities in the two molecules are equal in magnitude, although individual components in the $L M$ plane differ for the ring and nitro group since $L$ and $M$ are not symmetry axes for these groups in mNA.

We have therefore explored the origin dependence of the group polarizabilities, as detailed in the Appendix. We find that as a function of the chosen group origin the ratio of corresponding polarizability components in mNA and pNA varies rather little: for the charge-transfer component $\alpha_{q L}^{\mathrm{NH}_{2}, \mathrm{NH}_{2}}$ the ratio can never be forced to become unity, whereas for the local component $\alpha_{L L}^{\mathrm{NH}_{2}, \mathrm{NH}_{2}}$ it adopts the value unity for a wide range of origins. Given the already encouraging agreement between corresponding components of the group generalized polarizabilities in the molecular axes, the improved transferability obtainable by this refinement does not seem worth the additional effort, and so we have not pursued it farther.

In conclusion, expressing the polarizability response of a molecule in terms of functional group contributions reduces substantially the number of distributed polarizability components. It provides an approach compatible with chemical insights, and is useful for investigating the transferability of group polarizabilities between isomeric molecules. The functional group approach is less fine-grained than the atomic approach but retains all the features of distributed polariz- abilities (including the ability to treat response in nonuniform fields), and hence could be helpful in applying TPEP to force fields for molecular dynamics simulations.

\section{ACKNOWLEDGMENTS}

M.ihP. would like to thank Professor W. J. Blau for providing computational facilities and S. O'Flaherty for assistance with mNA calculations.

\section{APPENDIX: TRANSFORMATION OF GROUP POLARIZABILITIES TO LOCAL ORIGINS}

We begin by considering the group dipole moments. In order to simplify our expressions, we describe the charge distribution within functional group $F$ not by a continuous charge density but by a discrete set of charges $q_{i}^{F}$ at positions $\mathbf{r}_{i}^{F}$, so that the group dipole moment can be written as

$$
\boldsymbol{\mu}^{F}=\sum_{i} \mathbf{r}_{i}^{F} q_{i}^{F} .
$$

If the origin is moved to $\mathbf{R}$, the dipole moment becomes

$$
\mu^{\prime F}=\sum_{i}\left(\mathbf{r}_{i}^{F}-\mathbf{R}\right) q_{i}^{F}=\mu^{F}-\mathbf{R} q^{F},
$$

where $q^{F}$ is the net charge on group $F$, so that the dipole moment of the group depends on the charge it carries and on the choice of origin. (However, the total molecular dipole moment does not depend on the choice of origin provided the molecule is neutral, i.e., that the $q^{F}$ sum to zero.) The origin dependence of the group dipole moment is relevant to the question of determining potentially transferable group moments, which entails extracting group moments from different molecules and examining how far they are independent of the molecule in which they are located. This is straightforward, at least in principle, if each group can be treated as carrying zero net charge. However, once a group carries a net charge, the issue becomes more complicated. Even when the net charge is small, the charge-transfer contribution can be significant if the group is situated at an extremity of the molecule so that the charge is transferred a long distance. One could then decompose the calculated group dipole moment into the "local" group dipole moment that arises from the group dipole moment relative to some origin defined in the group and the "charge-transfer" contribution that arises from the group net charge and the distance of the group origin from the molecular origin. The local group dipole moment offers the possibility of being transferable, and in particular reduces to a unique origin-independent group dipole moment when the group net charge is zero, as here when charge flow is suppressed.

This analysis can now be extended to explore the origin dependence of the polarizabilities. The group dipole moment $\boldsymbol{\mu}^{\prime F}$ given by Eq. (A2) varies with a field $\mathbf{X}^{G}$ at group $G$ according to

$$
\partial \boldsymbol{\mu}^{\prime F} / \partial \mathbf{X}^{G}=\partial \boldsymbol{\mu}^{F} / \partial \mathbf{X}^{G}-\mathbf{R} \partial q^{F} / \partial \mathbf{X}^{G} .
$$

Taking $\mathbf{X}^{G}$ as the potential or the electric field leads to two variants of Eq. (A3) for group generalized polarizabilities 


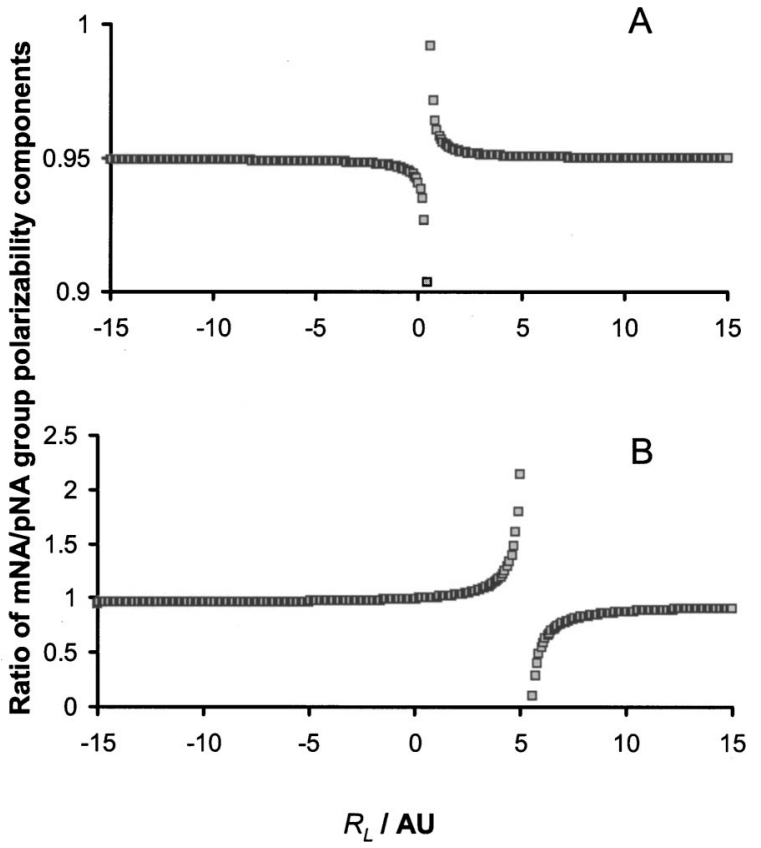

FIG. 2. Ratio of amino group polarizability contributions (mNA:pNA) as a function of $R_{L}$, the $L$ axis component of the displacement $\mathbf{R}$ of the group

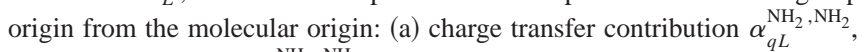
(b) local contribution $\alpha_{L L}^{\mathrm{NH}_{2}, \mathrm{NH}_{2}}$.

$$
\alpha_{\alpha q}^{\prime F G}=\alpha_{\alpha q}^{F G}-R_{\alpha} \alpha_{q q}^{F G}
$$

and

$$
\alpha_{\alpha \beta}^{\prime F G}=\alpha_{\alpha \beta}^{F G}-R_{\alpha} \alpha_{q \beta}^{F G} .
$$

By means of these equations, the group polarizabilities can be decomposed into charge, transfer and local contributions, as with the group dipole moments. The local contributions can also be transformed to a group axis system in order to remove dependence on the choice of molecular axis system.

The similarity of pNA and mNA group responses indicates that the local group polarizabilities can be transferable when charge flow is suppressed (Table III), but not once charge flow is allowed (Table II), because charge-transfer contributions can never be completely transferable. On the other hand, the group generalized polarizabilities that treat charge transfer explicitly are more transferable. We have explored whether this potential for transferability can be taken farther by using Eqs. (A4) and (A5) to calculate the chargetransfer and local generalized polarizability contributions for pNA and mNA as a function of the displacement $\mathbf{R}$ of the group origin from the molecular origin; $\mathbf{R}=0$ corresponds to the results in Tables IV and V. Figure 2 shows results for the simplest case of the amino group, where the group axes and the molecular axes coincide in both molecules, with the group origin displaced along the $L$ symmetry axis. For a suitable choice of $R_{L}$, the ratio of the charge-transfer contributions $\alpha_{q L}^{\mathrm{NH}_{2}, \mathrm{NH}_{2}}$ for the two molecules varies very little from 0.95 except around $R_{L}=0$, where it diverges, whereas the ratio of the local contributions $\alpha_{L L}^{\mathrm{NH}_{2}, \mathrm{NH}_{2}}$ varies rather little from unity except around $R_{L}=5$ a.u., where it too diverges. Similar results are obtained for the $\mathrm{C}_{6} \mathrm{H}_{4}$ and $\mathrm{NO}_{2}$ groups.

${ }^{1}$ A. J. Stone, Phys. Lett. 83A, 233 (1983).

${ }^{2}$ A. J. Stone, Mol. Phys. 56, 1065 (1985).

${ }^{3}$ R. F. W. Bader, Atoms in Molecules-A Quantum Theory (Oxford University Press, Oxford, 1990)

${ }^{4}$ P. L. A. Popelier, Atoms in Molecules-An Introduction (Pearson Education, Harlow, 2000)

${ }^{5}$ G. Jansen, C. Hättig, B. A. Heß, and J. G. Ángyán, Mol. Phys. 88, 69 (1996)

${ }^{6}$ C. Hättig, G. Jansen, B. A. Heß, and J. G. Ángyán, Mol. Phys. 91, 145 (1997).

${ }^{7}$ A. J. Stone, C. Hättig, G. Jansen, and J. G. Ángyán, Mol. Phys. 89, 595 (1996).

${ }^{8}$ C. Chipot, J. G. Ángyán, and C. Millot, Mol. Phys. 94, 881 (1998).

${ }^{9}$ J. Ángyán, G. Jensen, M. Loos, C. Hättig, and B. A. Heß, Chem. Phys. Lett. 219, 267 (1994)

${ }^{10}$ H. Reis, M. G. Papadopoulos, C. Hättig, J. Ángyán, and R. W. Munn, J. Chem. Phys. 112, 6161 (2000).

${ }^{11}$ M. in het Panhuis, P. L. A. Popelier, R. W. Munn, and J. Ángyán, J. Chem. Phys. 114, 7951 (2001).

${ }^{12}$ A. Aviram and M. A. Ratner, Chem. Phys. Lett. 29, 277 (1974).

${ }^{13}$ R. M. Metzger, Acc. Chem. Res. 32, 950 (1999).

${ }^{14}$ C. Kergueris, J. P. Bourguin, S. Palacin, D. Estève, C. Urbina, M. Magoga, and C. Joachim, Phys. Rev. B 59, 12505 (1999).

${ }^{15}$ S. N. Yaliraki, A. E. Roitberg, C. Gonzalez, V. Mujica, and M. A. Ratner, J. Chem. Phys. 111, 6997 (1999).

${ }^{16}$ L. E. Hall, J. R. Reimers, N. S. Hush, and K. Silverbrook, J. Chem. Phys. 112, 1510 (2000).

${ }^{17}$ M. in het Panhuis, R. W. Munn, P. L. A. Popelier, J. N. Coleman, B. Foley, and W. J. Blau, Proc. Natl. Acad. Sci. U.S.A. 99, 6514 (2002).

${ }^{18}$ Yu. A. Abramov, A. V. Volkov, and P. Coppens, Chem. Phys. Lett. 311, 81 (1999).

${ }^{19}$ C.-B. Park, U.-S. Choi, and C.-J. Kim, Synth. Met. 71, 1701 (1995).

${ }^{20}$ C. Daniel and M. Dupuis, Chem. Phys. Lett. 171, 209 (1990).

${ }^{21}$ H. Reis, M. G. Papadopoulos, P. Calaminici, K. Jug, and A. M. Köster, Chem. Phys. 261, 359 (2000).

${ }^{22}$ MORPHY01, a program written by P. L. A. Popelier with a contribution from R. G. A. Bone, D. Kosov, and M. in het Panhuis, UMIST, Manchester, UK, 2000

${ }^{23}$ B. Champagne, Chem. Phys. Lett. 261, 57 (1996).

${ }^{24}$ T. Shoda and D. Williams, J. Mol. Struct.: THEOCHEM 357, 1 (1995).

${ }^{25}$ A. C. Skapski and J. L. Stevenson, J. Chem. Soc., Perkin Trans. 2 1973, 1197 (1973)

${ }^{26}$ D. R. Borst, T. M. Korter, and D. W. Pratt, Chem. Phys. Lett. 350, 485 (2001).

${ }^{27}$ B. H. Cardelino, C. E. Moore, and R. E. Stickel, J. Phys. Chem. 95, 8645 (1991)

${ }^{28}$ C. R. Le Sueur and A. J. Stone, Mol. Phys. 83, 293 (1994).

${ }^{29}$ K. E. Laidig and R. F. W. Bader, J. Chem. Phys. 93, 7213 (1990).

${ }^{30}$ R. F. W. Bader and C. F. Matta, Int. J. Quantum Chem. 85, 592 (2001). 